\title{
Decidable and Undecidable Problems in Schedulability Analysis Using Timed Automata*
}

\author{
Pavel Krčál and Wang Yi \\ Uppsala University \\ Department of Information Technology \\ P.O. Box 337, S-751 05 Uppsala, Sweden \\ \{pavelk, yi\}@it.uu.se
}

\begin{abstract}
We study schedulability problems of timed systems with nonuniformly recurring computation tasks. Assume a set of real time tasks whose best and worst execution times, and deadlines are known. We use timed automata to describe the arrival patterns (and release times) of tasks. From the literature, it is known that the schedulability problem for a large class of such systems is decidable and can be checked efficiently.

In this paper, we provide a summary on what is decidable and what is undecidable in schedulability analysis using timed automata. Our main technical contribution is that the schedulability problem will be undecidable if these three conditions hold: (1) the execution times of tasks are intervals, (2) a task can announce its completion time, and (3) a task can preempt another task. We show that if one of the above three conditions is dropped, the problem will be decidable. Thus our result can be used as an indication in identifying classes of timed systems that can be analysed efficiently.
\end{abstract}

\section{Introduction}

Timed automata AD94 has been developed as a basic semantic model for real time systems. Recently it has been applied to solve scheduling problems, such as job-shop scheduling AM01AM02 Abd02 Feh02 HLP01 and real time scheduling MV94FPY02 $\mathrm{AFM}^{+} 02$ FMPY03WH03. The basic idea behind these works is to model real time tasks (or jobs) and scheduling strategies of a system as variants of timed automata and then check the reachability of pre-specified states. As the reachability problem of such automata is decidable, the scheduling problems can be solved automatically and in many cases efficiently (e.g. for fixed priority scheduling [FMPY03]) using a model-checker such as Kronos [Yov97, UPPAAL LPY97] or HYTECH HHWT97. For preemptive scheduling, stop-watch automata have been used to model preemption [MV94CL00 Cor94AM02]. But since the reachability problem for this class of

* This work has been partially supported by the Swedish Research Council and the European Research Training Network GAMES. 
automata is undecidable $\left.\mathrm{ACH}^{+} 95\right]$ there is no guarantee for termination in the general case.

We adopt the model presented in EWY98]. The essential idea behind the model is to use a timed automaton (control automaton) to describe the release (or arrival) patterns of the tasks. Tasks are released when control automaton makes a discrete transition. Each task has specified its computation (execution) time and its deadline. Released tasks are stored in a task queue and executed on a processor according to a scheduling strategy. There is a straightforward translation of such a system into a timed automaton for non-preemptive scheduling strategies. For the preemptive case, the schedulability problem was suspected to be undecidable due to the nature of preemption that may need the power of stop-watch to model.

In the original work, tasks in the queue cannot send any information back to the control automaton. The only communication between the control automaton and the task queue is the release of tasks. Once a task has been released, the control automaton has no possibility to find out any information about its execution. In particular, the control automaton does not know whether a task has finished or not. The behaviour of the control automaton is independent from the state of the queue. We say that this is a system with one-way communication. In this paper, we study systems where tasks can tell the control automaton that their execution has been just finished (systems with two-way communication). As an illustration, consider a company, where the boss assigns jobs to employees from time to time. In the model with one-way communication, the employees do not tell the boss when they finish their jobs whereas in the model with two-way communication they do.

The execution time of a task can vary within an interval - only the best and the worst execution time of each task is known. This is a natural assumption for modeling, because in many cases we cannot establish the exact computation time of a task (it depends on many circumstances from which the model abstracts), but we can establish some bounds for it. In the schedulability analysis of the systems with two-way communication we have to consider each possible finishing time, because it can influence the future behaviour of the control automaton. For instance, the boss can become too optimistic when several employees finish their jobs quickly and he can assign too many jobs to other employees. Or on the other hand, the boss can assign fewer jobs (or stop assigning new jobs at all) when the processing of some old one takes too much time (e.g. exception handling).

Recent results [FPY02 FMPY03] show that some of the schedulability problems related to preemptive scheduling are decidable. The decidability of the schedulability analysis has been proven for the following models. In FPY02] only systems with one-way communication are considered. However, the execution times of tasks can be intervals though it is not stated clearly in this work. The best execution time is not important for the schedulability analysis of the systems with one-way communication. In FMPY03 tasks can update data variables shared between them and the control automaton upon their completion (system with 
two-way communication), but the computation time should be a known constant for each task. The natural question is if schedulability analysis remains decidable for systems with two-way communication and interval execution times.

Unfortunately, the answer is negative. As the main technical contribution of this paper, we show that (1) the interval execution time of tasks, (2) the ability of the control automaton to test the exact completion time of tasks, and (3) preemption are sufficient and necessary to code the halting problem for twocounter machines. We shall also summarise previous decidability results and discuss other variants of the problem. Our goal is to identify as closely as possible the borderline between decidable and undecidable problems in schedulability analysis using timed automata. Hopefully, our result can be used as an indication in determining which classes of real-time models can be analysed efficiently.

The rest of the paper is organised as follows. In Section 2 we formally introduce our model, define the schedulability problem, and summarise previous decidability results. Section 3 contains the undecidability proof of the schedulability problem for our model. We discuss several variants of this model in Section 4 Section 5 concludes the paper with a summary and future work.

\section{Preliminaries}

\subsection{Timed Automata with Tasks}

To model two-way communication, we assume that each automaton has a distinguished clock, that is reset whenever a task finishes. This allows each task to announce its completion to the automaton. We have chosen reseting of the clock because even this simple model of two-way communication between tasks and the control automaton is sufficient for encoding of the two-counter machine. Other models of two-way communication, such as updating shared data variables upon completion are discussed later.

Syntax. Let $\mathcal{P}$ ranged over by $P, Q$ denote a finite set of task types. A task type may have different instances that are copies of the same program with different inputs. Each task $P$ is characterised as a pair $([B, W], D)$, where $[B, W]$ is the execution time interval and $D$ is the deadline for $P, B \leq W \leq D \in \mathcal{N}_{0}$ and $W \neq 0$. The deadline $D$ is relative, meaning that when task $\bar{P}$ is released, it should finish within $D$ time units. We use $B(P), W(P)$, and $D(P)$ to denote the best execution time, the worst execution time, and the relative deadline of the task $P$.

As in timed automata, assume a finite set of real-valued variables $\mathcal{C}$ for clocks. We use $\mathcal{B}(\mathcal{C})$ ranged over by $g$ to denote the set of conjunctive formulas of atomic constraints in the form: $a \sim N$ or $a-b \sim M$ where $a, b \in \mathcal{C}$ are clocks, $\sim \in\{\leq,<, \geq,>\}$, and $N, M$ are natural numbers. We use $\mathcal{B}_{I}(\mathcal{C})$ for the subset of $\mathcal{B}(\mathcal{C})$ where all atomic constraints are of the form $a \sim N$ and $\sim \in\{<, \leq\}$. The elements of $\mathcal{B}(\mathcal{C})$ are called clock constraints or guards. 
Definition 1. A timed automaton extended with tasks, over clocks $\mathcal{C}$ and tasks $\mathcal{P}$ is a tuple $\left\langle N, l_{0}, E, I, M\right.$, check $\rangle$ where

$-\left\langle N, l_{0}, E, I\right\rangle$ is a timed automaton where

- $N$ is a finite set of locations,

- $l_{0} \in N$ is the initial location,

- $E \subseteq N \times \mathcal{B}(\mathcal{C}) \times 2^{\mathcal{C}} \times N$ is the set of edges, and

- $I: N \mapsto \mathcal{B}_{I}(\mathcal{C})$ is a function assigning each location with a clock constraint (a location invariant),

- $M: N \hookrightarrow \mathcal{P}$ is a partial function assigning locations with a task type 1 and

- check $\in \mathcal{C}$ is the clock which is reset whenever a task finishes.

As a simplification we will use $l \stackrel{g, r}{\longrightarrow} m$ to denote $(l, g, r, m) \in E$.

For convenience, in the rest of the paper we use extended timed automata (ETA) or simply automata when it is understood from the context instead of timed automata extended with tasks.

Operational Semantics. Extended timed automata may perform two types of transitions just as standard timed automata. Intuitively, a discrete transition in an automaton denotes an event triggering a task. Whenever a task is released, it will be put in a scheduling (or task) queue for execution. A delay transition corresponds to the execution of the running task with the highest priority and idling for the other tasks waiting to run.

We represent the values of clocks as functions (called clock assignments) from $\mathcal{C}$ to the non-negative reals. A state of an automaton is a triple $(l, \sigma, q)$ where $l$ is the current control location, $\sigma$ the clock assignment, and $q$ is the current task queue. We assume that the task queue takes the form: $\left[P_{1}\left(b_{1}, w_{1}, d_{1}\right), \ldots\right.$, $\left.P_{n}\left(b_{n}, w_{n}, d_{n}\right)\right]$ where $P_{i}\left(b_{i}, w_{i}, d_{i}\right)$ denotes a released instance of task type $P_{i}$ with remaining best computing time $b_{i}$, remaining worst computing time $w_{i}$, and relative deadline $d_{i}$ denoted by $b\left(P_{i}\right), w\left(P_{i}\right)$ and $d\left(P_{i}\right)$ respectively.

A queue reordering function is a sorting function which changes the ordering of the task queue elements according to the task types and parameters. It takes a task queue as an input and returns a task queue with the unmodified tasks that may be sorted in a different order. A scheduling strategy (Sch) is a queue reordering function which changes the ordering of task types only, but never changes the ordering of task instances of the same type. A non-preemptive strategy will never change the position of the first element in a queue, whereas a preemptive scheduling strategy may change the position of the first element in the queue (the one which is currently executed). E.g. FPS (fixed priority scheduling) or EDF (earliest deadline first) are preemptive scheduling strategies. For example, $\operatorname{EDF}([P(3.1,4.9,10), Q(4,4.5,5.3))]=[Q(4,4.5,5.3), P(3.1,4.9,10))]$.

${ }^{1}$ Note that $M$ is a partial function meaning that some of the locations may have no task. 
Run is a function which given a real number $t$ and a task queue $q$ returns the task queue after $t$ time units of execution on a processor. The result of $\operatorname{Run}(q, t)$ for $q=\left[P_{1}\left(b_{1}, w_{1}, d_{1}\right), P_{2}\left(b_{2}, w_{2}, d_{2}\right), \ldots, P_{n}\left(b_{n}, w_{n}, d_{n}\right)\right]$ is defined as $q^{\prime}=\left[P_{1}\left(b_{1}-\right.\right.$ $\left.\left.t, w_{1}-t, d_{1}-t\right), P_{2}\left(b_{2}, w_{2}, d_{2}-t\right), \ldots, P_{n}\left(b_{n}, w_{n}, d_{n}-t\right)\right]$. For example, let $q=$ $[Q(2,3,5), P(4,7,10)]$. Then $\operatorname{Run}(q, 3)=[Q(-1,0,2), P(4,7,7)]$ in which the first task has been executed for 3 time units (and it will be removed from the queue). A task $P$ in the queue may finish when $b(P)=0$ and $w(P) \geq 0$, and it must finish when $w(P)=0$. Finished tasks are removed from the queue.

Further, for a non-negative real number $t$, we use $\sigma+t$ to denote the clock assignment which maps each clock $a$ to the value $\sigma(a)+t, \sigma \models g$ to denote that the clock assignment $\sigma$ satisfies the constraint $g$ and $\sigma[r]$ for $r \subseteq \mathcal{C}$ to denote the clock assignment which maps each clock in $r$ to 0 and agrees with $\sigma$ for the other clocks (i.e. $\mathcal{C} \backslash r$ ). We omit braces when $r$ is a singleton.

Definition 2. Given a scheduling strategy Sch, the semantics of an extended timed automaton $\left\langle N, l_{0}, E, I, M\right.$, check $\rangle$ with initial state $\left(l_{0}, \sigma_{0}, q_{0}\right)$ is a transition system defined by the following rules:

$$
\begin{aligned}
& -(l, \sigma, q) \longmapsto \operatorname{Sch}(m, \sigma[r], \operatorname{Sch}(M(m):: q)) \text { if } l \stackrel{g, a, r}{\longrightarrow} m, \sigma \models g, \text { and } \sigma[r] \models I(m) \\
& -(l, \sigma,[]) \stackrel{t}{\longrightarrow} \operatorname{Sch}(l, \sigma+t,[]) \text { if }(\sigma+t) \models I(l) \\
& -(l, \sigma, P:: q) \stackrel{t}{\longrightarrow} \operatorname{sch}(l, \sigma+t, \operatorname{Run}(P:: q, t)) \text { if } t \leq w(P) \text { and }(\sigma+t) \models I(l) \\
& -(l, \sigma, P:: q) \stackrel{0}{\longrightarrow} \operatorname{Sch}(l, \sigma[\text { check }], q) \text { if } b(P) \leq 0 \leq w(P) \text { and } \sigma[\text { check }] \models I(l)
\end{aligned}
$$

where $P:: q$ denotes the queue with the process $P$ inserted in $q$ (at the first position), and [] denotes the empty queue.

\subsection{Schedulability and Decidability}

In this subsection we define the schedulability problem for ETA and give a summary of the previous decidability results. Undecidability is discussed in the following section. We first mention that we have the same notion of reachability as for ordinary timed automata.

Definition 3. We shall write $(l, \sigma, q) \longrightarrow \mathrm{Sch}\left(l^{\prime}, \sigma^{\prime}, q^{\prime}\right)$ if $(l, \sigma, q) \longmapsto \mathrm{Sch}\left(l^{\prime}, \sigma^{\prime}, q^{\prime}\right)$ or $(l, \sigma, q) \stackrel{t}{\longrightarrow} \mathrm{Sch}\left(l^{\prime}, \sigma^{\prime}, q^{\prime}\right)$ for a delay $t$. For an automaton with initial state $\left(l_{0}, \sigma_{0}, q_{0}\right)$ and for a scheduling strategy $\mathrm{Sch}$, we say that $(l, \sigma, q)$ is reachable iff $\left(l_{0}, \sigma_{0}, q_{0}\right)(\longrightarrow \mathrm{Sch}) *(l, \sigma, q)$.

Now we can formalise the notion of schedulability.

Definition 4. (Schedulability) A state $(l, \sigma, q)$ where $q=\left[P_{1}\left(b_{1}, w_{1}, d_{1}\right), \ldots\right.$, $\left.P_{n}\left(b_{n}, w_{n}, d_{n}\right)\right]$ is a failure denoted $\left(l, \sigma\right.$, Error) if there exists $i$ such that $w_{i} \geq 0$ and $d_{i}<0$, that is, a task failed in meeting its deadline. Naturally an automaton $A$ with initial state $\left(l_{0}, \sigma_{0}, q_{0}\right)$ is non-schedulable with Sch iff $(l, \sigma$, Error $)$ is 
reachable for some $l$ and $\sigma$. Otherwise, we say that $A$ is schedulable with Sch. 2 More generally, we say that $A$ is schedulable iff there exists a scheduling strategy Sch with which $A$ is schedulable.

The following decidability results apply to some simpler variants of the schedulability problem for extended timed automata. By this we show, that whenever preemption, clock resets, or interval execution times are not allowed, the problem becomes decidable.

Theorem 1 ([EWY98]). The problem of checking schedulability for extended timed automata with non-preemptive scheduling strategy is decidable.

Proof. The proof in EWY98 handles only tasks with worst case execution time and tasks without clock resets. But it can be easily modified for our model.

The schedulability problem is decidable even for preemptive scheduling strategies when tasks are not allowed to communicate with an automaton (to reset clocks). Execution times of tasks can be intervals.

Theorem 2 ([FPY02]). The problem of checking schedulability for extended timed automata with one-way communication (tasks do not reset clocks) is decidable.

Proof. It is easy to observe that we can consider only the worst case execution time of each task and the proof is given in [FPY02.

The schedulability is decidable even for extended timed automata with two-way communication (tasks may reset clocks by the end of their execution) when the computation time is a known constant for each task.

Theorem 3 ([FMPY03]). The problem of checking schedulability for extended timed automata is decidable if $B(P)=W(P)$ for all tasks $P$.

Proof. The proof is given in [FMPY03].

When tasks have interval execution time in the system with two-way communication the computation of the control automaton can be influenced by the exact completion time of a task. The control automaton can take into consideration if a task has already finished or not and can proceed to different locations in different cases. For example, if a task does not finish in a given time the machine stops (it deadlocks, or proceeds to some idle state, i.e. no new tasks are released, just the already released tasks are computed). By this we can cut off (e.g. deadlock) branches where a task had "wrong" computation time. In fact,

\footnotetext{
${ }^{2}$ Note that the state might not be failure denoted when ETA is deadlocked and time cannot progress, even if time flow would lead to a failure denoted state. Therefore, a model where such states are reachable might still be schedulable. This is not fundamental for our proof and these states could be prohibited. But it would induce some technical difficulties making the proof less clear.
} 
we can construct an automaton which proceeds to a certain location if and only if a task has been executed for some given exact (real) time.

Preemption enables us to sum up (accumulate) running times of tasks. Response time of the preempted task is increased by running time of each preempting task. This is sufficient to encode the two-counter machine.

\section{Undecidability}

Our main result in this paper is that the schedulability problem with fixed priority scheduling strategy for the automata defined in the previous section is undecidable. However, the proof does not depend on fixed priority scheduling strategy and it can be easily modified for almost all preemptive scheduling strategies (e.g. the proof holds for EDF without any modification).

Theorem 4. The problem of checking whether extended timed automaton (defined in Definition 1) is schedulable with fixed priority scheduling strategy is undecidable.

The proof is done by reduction of the halting problem for two-counter machine to the schedulability problem for ETA. A two-counter machine consists of a finite state control unit and two unbounded non-negative integer counters. Initially, both counters contain the value 0 . Such a machine can execute three types of instructions: incrementation of a counter, decrementation of a counter, and branching based upon whether a specific counter contains the value 0 . Note that decrementation of a counter with the value 0 leaves this counter unchanged. After execution of an instruction, a machine changes deterministically its state. One state of a two-counter machine is distinguished as halt state. A machine halts if and only if it reaches this state.

We present an encoding of a two-counter machine $M$ using extended timed automaton $\mathcal{A}_{M}$ such that $M$ halts if and only if $\mathcal{A}_{M}$ is non-schedulable, based on the undecidability proofs of [HKPV98. In the construction, the states of $M$ correspond to specific locations of $\mathcal{A}_{M}$ and each counter is encoded by a clock. We shall show how to simulate the two-counter machine operations. First, we adopt the notion of W-wrapping of [HKPV98].

Definition 5. An extended timed automaton over set of clocks $\mathcal{C}$ is $\mathrm{W}$-wrapping if for all states $(l, \sigma, q)$ reachable from its initial state and for all clocks $c \in \mathcal{C}$ : $\sigma \models c \leq W$. A W-wrapping edge for a clock $c$ and a location $l$ is an edge from $l$ to itself that is labeled with the guard $c=W$ and which resets the clock $c$. A clock that is reset only by wrapping edges is called system clock 3 Each time period between two consecutive time points at which any system clock contains value 0 is called $\mathrm{W}$-wrapping period.

\footnotetext{
${ }^{3}$ Note that all system clocks contain the same value.
} 
We use wrapping to simulate discrete steps of two-counter machine. Each step is modeled by several $\mathrm{W}$-wrapping periods. We define the wrapping-value of a clock to be the value of the clock when the system clock is 0 . Note that a clock is carrying the same wrapping value if it is not reset by another edge than the wrapping edges. This principle is shown in Figure 1] where $a$ is a system clock and clock $t$ contains the same wrapping-value when the automaton takes transitions $e_{1}$ and $e_{3}$.
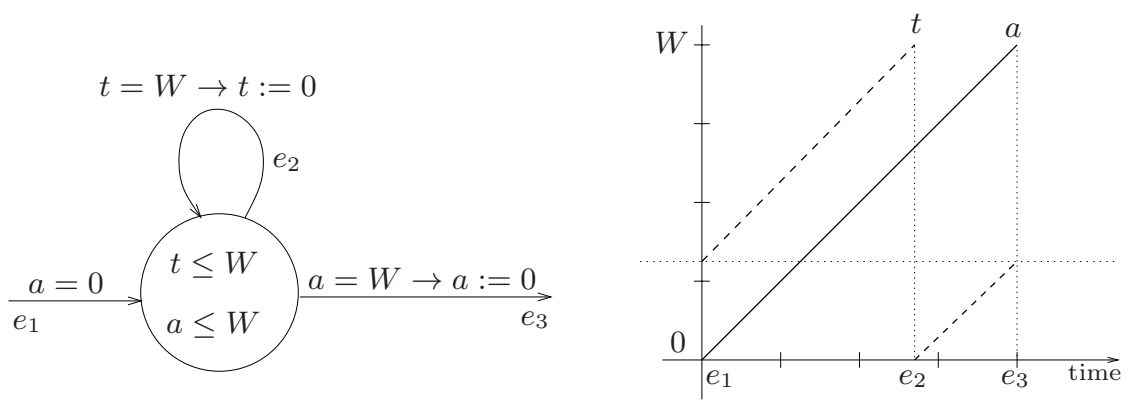

Fig. 1. The wrapping edge $e_{2}$ makes clock $t$ carry the same wrapping-value when the transitions $e_{1}$ and $e_{3}$ are taken.

We shall encode a two-counter machine $M$ with counters $C$ and $D$ using a 4wrapping automaton $\mathcal{A}_{M}$ with one system clock denoted $a$ and five other clocks $c, d, h, t$ and check. In particular, we encode counters $C$ and $D$ of $M$ by clocks $c$ and $d$ like this: counter value $v$ corresponds to the clock wrapping-value $2^{1-v}$. We use the density of the continuous domain to encode arbitrarily large values of the counters. Decrementation (incrementation) of a counter corresponds to doubling (halving) the wrapping-value of the corresponding clock. Test for zero corresponds to the check whether the clock wrapping-value equals to 2 .

Now we show how to simulate the decrementation operation by doubling the wrapping-value of the clock $d$. To do this, we use two tasks: short and long. The task short has execution time within interval $[0,1]$ and deadline 50; the task long has execution time within interval $[8,8]$ and deadline 100 . The tasks reset clock check by the end of their execution. Moreover, the priority of short is higher than the priority of long, i.e. short always preempts long. Notice that the execution time of task short can vary and the execution time of the task long is fixed.

The basic idea of doubling a wrapping-value $v \in(0,1]$ of clock $d$ is as follows: we assume that the current wrapping-value of $d$ is $v$. We copy it to clock $t$ (that is, to make the wrapping-value of clock $t$ to be $v$ ). We release the task long nondeterministically and reset $d$. The idea is to use $d$ to record the response time for long. We release two instances of short before long finishes, that is preempt long twice by short. We make sure that the execution time of each of these two 


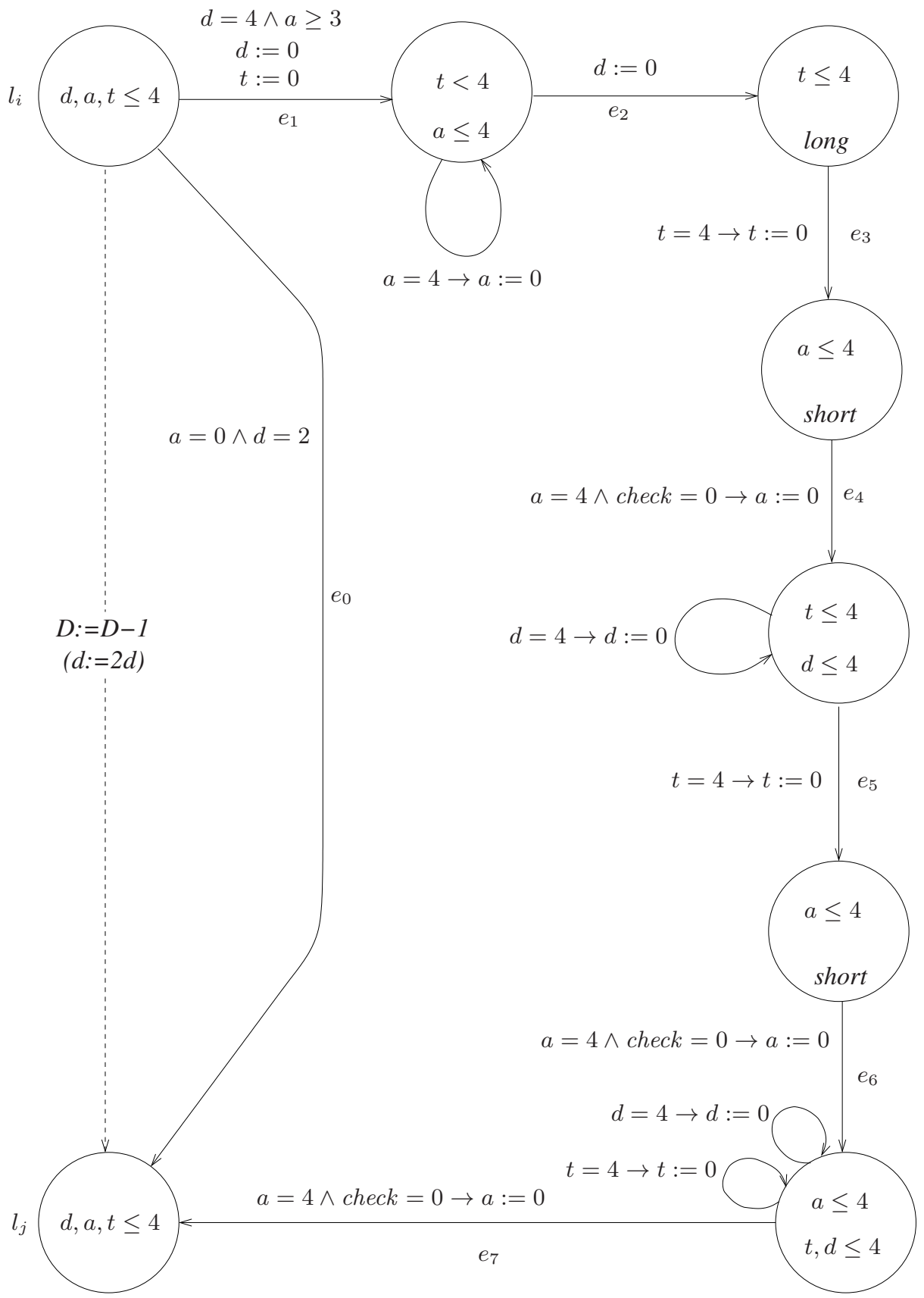

Fig. 2. A part of reduction automaton corresponding to a decrementation of $D$. The wrapping edges for clocks $c, h$, check, and for all clocks in locations $l_{i}, l_{j}$ are omitted. The location invariants $c \leq 4, h \leq 4$, and check $\leq 4$ are also omitted. 


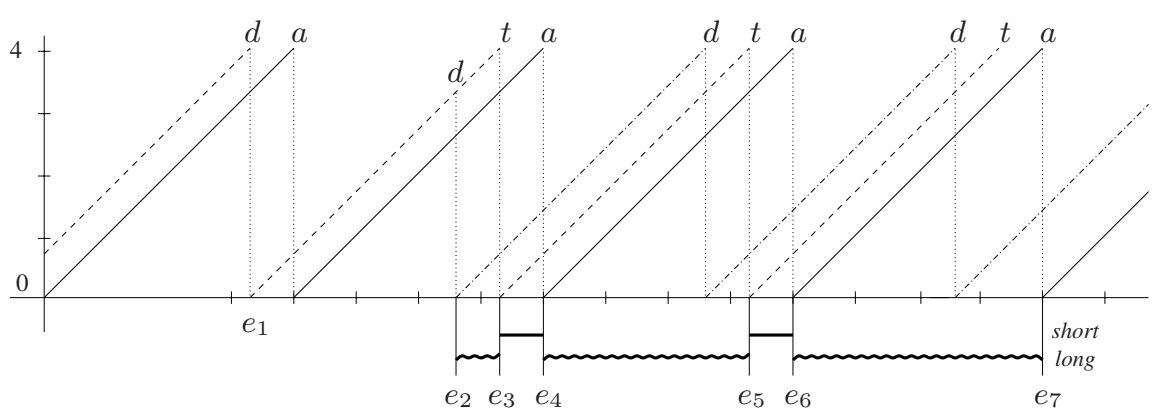

Fig. 3. Time chart of the doubling procedure.

instances of short is exactly $v$ time units. Note that $v$ can be any real number within the interval $(0,1]$. Then the response time for long is exactly $8+2 v$. Note that if long finishes at a time point when the system clock $a$ is reset to 0 , the wrapping-value of $d$ is $2 v$. As long is released non-deterministically, there will be surely one such computation.

In Figure 2, we show the part of $\mathcal{A}_{M}$ that doubles the wrapping-value of clock d. Figure 3 illustrates the time chart of the doubling process. Assume that a two-counter machine $M$ is currently in state $s_{i}$ and that it wants to decrease the counter $D$ and then move to state $s_{j}$. The locations $l_{i}$ and $l_{j}$ of $\mathcal{A}_{M}$ correspond to the states $s_{i}$ and $s_{j}$ respectively. Note that the dashed edge shows the transition of the two-counter machine (it is not a transition of $\mathcal{A}_{M}$ ). Note also that the decrementation operation leaves a counter with value 0 unchanged; the automaton can move from $l_{i}$ directly to $l_{j}$ through the transition $e_{0}$ when $d$ contains the wrapping-value 2 (which corresponds to the counter value 0 ). Otherwise, the following steps are taken to double the wrapping-value of $d$.

Firstly, the wrapping-value of $d$ is copied to clock $t$ (by transition $e_{1}$ ), that is, $t$ carries the same wrapping-value as $d$. Then the automaton non-deterministically guesses the doubled wrapping-value of $d$ (note that when $d$ is reset, it will carry a new wrapping-value). It resets $d$ at nondeterministically chosen time instant and at the same time it releases the task long (transition $e_{2}$ ).

The automaton waits until clock $t$ reaches time 4 , then resets $t$ and releases short (transition $e_{3}$ ), which preempts long. Note that the wrapping-value of $t$ will remain to be $v$ and at this time point the value of the system clock $a$ is $4-v$. Therefore $a$ will reach 4 in $v$ time units.

The next transition $e_{4}$ is guarded by two constraints: $a=4$, check $=0$. To satisfy these constraints, the automaton has to wait in this location for $v$ time units, and task short must finish at this time point, which resets the clock check 4

${ }^{4}$ We have to make sure that check is not reset by a wrapping edge when it is tested by a guard of the automaton. This causes no technical difficulties and it is omitted from Figure 2 . 
By this we make short run (and prevent long from running) exactly for $v$ time units. Now we repeat this procedure again. That is, the automaton waits until $t=4$. Then it releases the task short and forces it to run exactly for $v$ time units (transitions $e_{5}$ and $e_{6}$ ).

Now, if the non-deterministic guess of the doubled wrapping-value of $d$ was correct, task long must finish when $a=4$, which makes the guard on $e_{7}$ become true and the automaton moves to location $l_{j}$.

So if the location $l_{j}$ is reachable, the wrapping-value of $d$ is $2 v$. This is stated in the following lemma.

Lemma 1. Let $\left(l_{i}, \sigma, q\right)$ be an arbitrary state of the automaton shown in Figure 2 where $\sigma(d)=v$ and $v \in(0,1]$, and $q$ is empty. Then $\left(l_{j}, \sigma^{\prime}, q^{\prime}\right)$ is reachable for some $\sigma^{\prime}$ and $q^{\prime}$ and if $\left(l_{j}, \sigma^{\prime}, q^{\prime}\right)$ is reachable, it must be the case that $q^{\prime}=[]$, and $\sigma^{\prime}(d)=2 v$.

Proof. The proof is obvious from the construction in Figure 2.

To increment a counter we need to halve a wrapping-value of a clock, say $c$. For this, we use the clock $h$ to copy the wrapping-value of $c$. The new wrapping-value $v$ of $c$ is nondeterministically guessed and it is checked by the above doubling procedure. If the wrapping-value of $h$ (the original wrapping-value of $c$ ) is $2 v$, then the automaton can proceed to the location corresponding to the destination state in an increment instruction.

To simulate branching, we construct two transitions outgoing from a location with guards $a=0 \wedge c=2$ and $a=0 \wedge c \neq 2$. The initial state of $M$ corresponds to a location where both $c$ and $d$ contain the wrapping-value 2 . This can be achieved by integer guards and resets.

The halt state corresponds to the location halt with unguarded self-loop releasing the task long whenever it is visited. It follows that the automaton $\mathcal{A}_{M}$ is schedulable if and only if the location halt is unreachable, i.e. the two-counter machine $M$ does not halt.

\section{Variants of the Problem}

The proof can be easily modified even for some variants of the original setting. By this we want to show that the only sufficient conditions for undecidability are the following: the execution times of tasks are within intervals, an automaton can test the exact completion time of tasks, and one task can be preempted by another one.

The schedulability problem is undecidable if we use data variables to model twoway communication as described in FMPY03 instead of a distinguished clock. Tasks can assign values to the variables shared between them and the control automaton. The automaton can use these variables in assignments and guards. 
In our construction, tasks assign the value 1 to the data variable $A$ upon completion. The edges $e_{4}$ and $e_{6}$ in the original construction are substituted by the edges $e^{\prime}, e^{\prime \prime}$ and by the location $l_{\text {int }}$ from Figure 4 The location where the task short is released is left exactly after $t$ time units, but short is not finished yet $(A=0)$. However, the time does not flow in the location $l_{\text {int }}$. Just the task short can finish here. When this happens the edge $e^{\prime \prime}$ becomes enabled and the automaton can proceed. In fact, an automaton $\mathcal{A}_{M}$ uses interleaving at the time instant when a task finishes to enforce and to measure correct execution time.

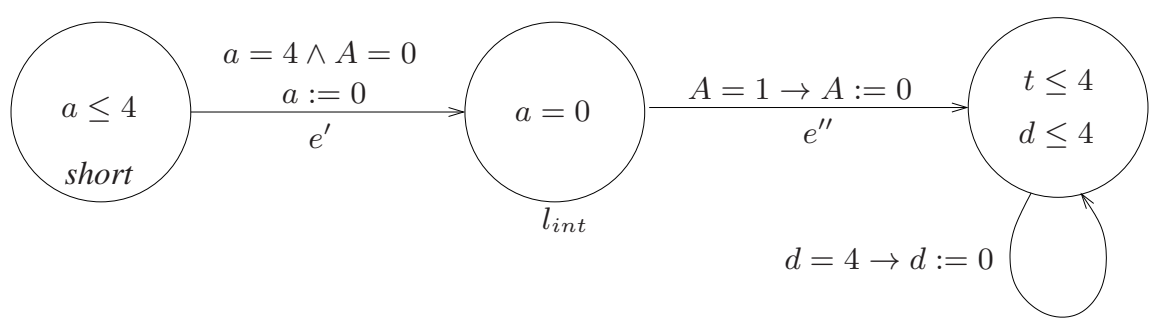

Fig. 4. Substitution of the clock resets by interleaving and data updates.

The schedulability problem is also undecidable if the tasks can only be released at integer time points. In this case, we encode a counter value $v$ as a clock wrappingvalue $4-2^{1-v}$. Decrementing (incrementing) of a counter does not correspond to doubling (halving) a clock wrapping-value anymore. Both instructions correspond to more complicated operations. Otherwise, the construction becomes even simpler. We do not need auxiliary clock $t$. Both long and short are released when $a=0$. The task short should finish when $d=0$ and we reset the clock $d$ to obtain the doubled wrapping-value when the task long finishes. Synchronisation can be forced either by clock resets or by data variable updates. Figure 5 shows the time chart of the doubling procedure.

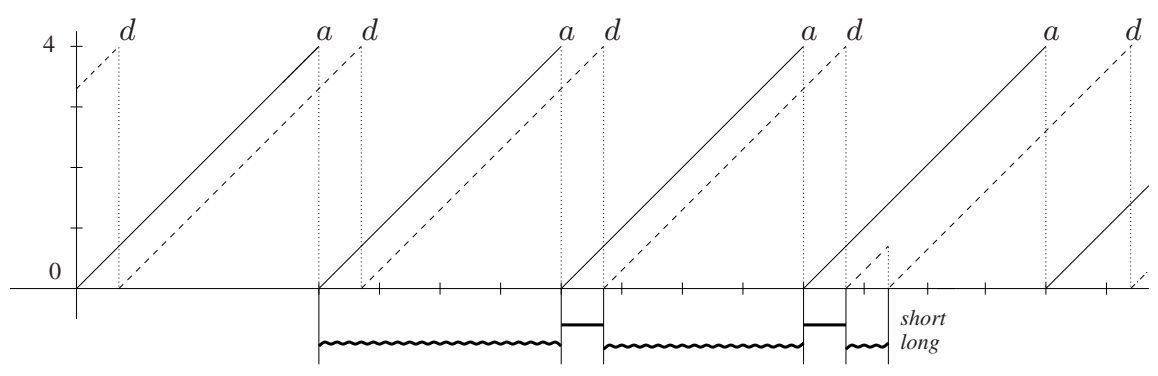

Fig. 5. The time chart of the doubling procedure for integer release points. 
Schedulability will also remain undecidable if we prohibit $B(P)=W(P)$, i.e. no task is allowed to have a constant computation time. Then we use the task $l_{\text {ong }} g_{1}$ with the execution interval $[7,8]$ instead of long. The guessed wrapping-value of $d$ can be less or equal to the correctly doubled value, because the task long can finish sooner. However, we repeat the whole doubling procedure with the task $\operatorname{long}_{2}$ which has the execution interval $[8,9]$. Now the automaton does not guess new wrapping-value of $d$, but uses the wrapping-value from the previous step. Therefore, this verifying procedure can succeed only if the wrapping-value of $d$ was guessed correctly in the first doubling procedure.

It is sufficient to use just one preemption for doubling a clock value. Therefore, even for systems where each task can be preempted only once during its execution the schedulability problem turns out to be undecidable. Figure6 shows the time chart of the doubling procedure. The task short has the execution time within interval $[3,4]$ and the task long has the execution time within $[4,4]$. New doubled value is nondeterministically guessed and the guess is verified by the procedure.

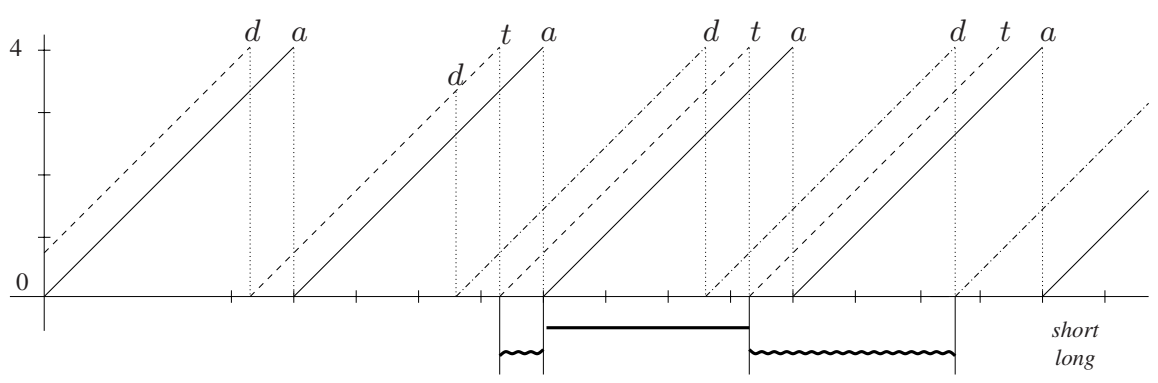

Fig. 6. Time chart of the doubling procedure using just one preemption.

Moreover, we present yet another variant of the extended timed automata for which we suspect the schedulability problem to be decidable.

Consider the extended timed automata with the following modification. The clock used for the task completion announcement (check) can appear only in the guards of the form $N \sim$ check $\sim M$ or $N \sim$ check where $\sim \in\{\leq,<\}, N, M \in \mathcal{N}$, and $N \neq M$. This means that we prohibit equality checking for this clock. The automaton can only decide upon whether the value of check lies in a non-singular interval. For this setting, it is an open problem whether the schedulability checking is decidable or not.

\section{Conclusions and Future Work}

We have studied timed systems where preemption can occur at any real time point. For these systems, the schedulability checking problem is decidable if 
either the computation time of each task is a known constant or the control automaton cannot test the exact completion time of the tasks. We have showed that the scheduling problem becomes undecidable if both of these restrictions are dropped. By comparing this result with known decidability results, we try to identify the borderline between decidable and undecidable problems in schedulability analysis for these systems.

As future work, we will try to identify a class of systems where only partial information about completion time of a task can be obtained by the control unit such that the schedulability problem will become decidable. We have presented a model of such class of systems in Section 4 as a candidate.

\section{References}

[Abd02] Y. Abdeddaïm. Scheduling with Timed Automata. PhD thesis, Verimag, 2002 .

$\left[\mathrm{ACH}^{+} 95\right]$ R. Alur, C. Courcoubetis, N. Halbwachs, T. A. Henzinger, P.-H. Ho, X. Nicollin, A. Olivero, J. Sifakis, and S. Yovine. The algorithmic analysis of hybrid systems. Theoretical Computer Science, 138(1):3-34, 1995.

[AD94] R. Alur and D. L. Dill. A theory of timed automata. Theoretical Computer Science, 126(2):183-235, 1994.

$\left[\mathrm{AFM}^{+} 02\right]$ T. Amnell, E. Fersman, L. Mokrushin, P. Pettersson, and W. Yi. Times a tool for modelling and implementation of embedded systems. In Proc. TACAS'02, volume 2280 of $L N C S$, pages 460-464. Springer, 2002.

[AM01] Y. Abdeddaïm and O. Maler. Job-shop scheduling using timed automata. In Proc. CAV'01, volume 2102 of LNCS, pages 478-492. Springer, 2001.

[AM02] Y. Abdeddaïm and O. Maler. Preemptive job-shop scheduling using stopwatch automata. In Proc. TACAS'02, volume 2280 of LNCS, pages 113126. Springer, 2002.

[CL00] F. Cassez and F. Laroussinie. Model-checking for hybrid systems by quotienting and constraints solving. In Proc. CAV'00, volume 1855 of LNCS, pages 373-388. Springer, 2000.

[Cor94] J. Corbett. Modeling and analysis of real-time ada tasking programs. In Proc. IEEE RTSS'94, pages 132-141, 1994.

[EWY98] C. Ericsson, A. Wall, and W. Yi. Timed automata as task models for event-driven systems. In Proceedings of Nordic Workshop on Programming Theory, 1998.

[Feh02] A. Fehnker. Citius, Vilius, Melius - Guiding and Cost-Optimality in Model Checking of Timed and Hybrid Systems. PhD thesis, KU Nijmegen, 2002.

[FMPY03] E. Fersman, L. Mokrushin, P. Pettersson, and W. Yi. Schedulability analysis using two clocks. In Proc. TACAS 2003, volume LNCS 2619, pages 224-239. Springer-Verlag, 2003.

[FPY02] E. Fersman, P. Pettersson, and W. Yi. Timed automata with asynchronous processes: Schedulability and decidability. In Proc. TACAS'02, volume 2280 of $L N C S$, pages 67-82. Springer, 2002.

[HHWT97] T.A. Henzinger, P.-H. Ho, and H. Wong-Toi. HYTECH: A model checker for hybrid systems. International Journal on Software Tools for Technology Transfer, 1(1-2):123-133, 1997. 
[HKPV98] T.A. Henzinger, P.W. Kopke, A. Puri, and P. Varaiya. What's decidable about hybrid automata? Journal of Computer and System Sciences, 57:94-124, 1998 .

[HLP01] T. Hune, K.G. Larsen, and P. Pettersson. Guided Synthesis of Control Programs using UpPAal. Nordic Journal of Computing, 8(1):43-64, 2001.

[LPY97] K.G. Larsen, P. Pettersson, and W. Yi. UPPAAL in a Nutshell. Int. Journal on Software Tools for Technology Transfer, 1(1-2):134-152, October 1997.

[MV94] J. McManis and P. Varaiya. Suspension automata: A decidable class of hybrid automata. In Proc. CAV'94, volume 818, pages 105-117. Springer, 1994.

[WH03] L. Waszniowski and Z. Hanzálek. Analysis of real time operating system based applications. In Proc. FORMATS'03, 2003.

[Yov97] S. Yovine. Kronos: A verification tool for real-time systems. International Journal on Software Tools for Technology Transfer, 1(1-2):110-122, 1997. 